\title{
Topological insulator metamaterials with tunable negative refractive index in the optical region
}

\author{
Tun Cao ${ }^{*}$ and Shuai Wang
}

\begin{abstract}
A blueshift tunable metamaterial (MM) exhibiting a double-negative refractive index based on a topological insulator (bismuth selenide, $\mathrm{Bi}_{2} \mathrm{Se}_{3}$ ) has been demonstrated in the near-infrared (NIR) spectral region. The potential of $\mathrm{Bi}_{2} \mathrm{Se}_{3}$ as a dielectric interlayer of the multilayer $\mathrm{MM}$ is explored. The optical response of elliptical nanohole arrays penetrating through $\mathrm{Au} / \mathrm{Bi}_{2} \mathrm{Se}_{3} / \mathrm{Au}$ films is numerically investigated using the finite difference time domain (FDTD) method. The blueshift tuning range of the MM is as high as $370 \mathrm{~nm}$ (from 2,140 to 1,770 nm) after switching the $\mathrm{Bi}_{2} \mathrm{Se}_{3}$ between its trigonal and orthorhombic states.
\end{abstract}

Keywords: Metamaterials; Metal-insulator-metal structures; Surface plasmons

PACS: 81.05.Xj; 73.40.Rw; 73.20.Mf

\section{Background}

Metamaterials (MMs) are artificially engineered composites that attract considerable interests due to their exceptional electromagnetic properties, which are not typically found in nature, such as negative refractive index and cloaking [1-4]. These MMs with various subwavelength resonant elements have offered magnetic and/or electric resonant responses to incident electromagnetic radiation, scalable from the microwave frequencies up to the terahertz and optical ones [5-7]. Particularly, nanohole resonators embedded in metal-dielectric-metal (MDM) multilayers are frequently used as building blocks of negative-refractive-index MMs [8-11], owing to the coupling between surface plasmons counterpropagating on the two closely spaced interfaces which results in a closed loop of the electric currents. This gives rise to magnetic dipolar resonances between the two coupled metal layers, while the continuous metallic strip parts provide the electric resonance moments [12,13]. All these features make the nanohole array perforating through MDM films become a strong candidate for developing three-dimensional negative-index MMs [14,15].

One of the obstacles in this progress is the resonance responses of MMs to the impinge light which are usually fixed once the dimension of the structure is determined,

\footnotetext{
* Correspondence: caotun1806@dlut.edu.cn

Department of Biomedical Engineering, Dalian University of Technology, Dalian 116024, People's Republic of China
}

thus making the MMs possess a limited bandwidth. However, for many applications (switching, modulation, filtering, etc.), it would be highly desirable to tune the MM resonances over a wide bandwidth. To this end, tunable photonic MMs, the spectral range of which can be controlled by changing the dielectric environment of the resonator with liquid crystals (LCs) [16-18]; phase transition materials [19,20]; and optical pumping [21,22] have been discussed recently. However, the challenge is to develop tunable MDM-MMs in the near-infrared (NIR) regime. It is due to the fact that frequency tunability of the MDMMM mainly requires for the interlayer dielectric material to possess a tunable effective dielectric constant in the NIR region, hence limiting the choice of the active materials. Here, we take a different approach to actively tune the resonant frequency of the MDM-MMs in the NIR regions by using bismuth selenide $\left(\mathrm{Bi}_{2} \mathrm{Se}_{3}\right)$ as the dielectric layer.

Recently, a rising Dirac material - topological insulators (TIs) - had been intensively researched in condensed matter physics $[23,24]$. In analogy to the optoelectronic applications of graphene, a thin layer of TIs has been theoretically predicted to be a promising material for broadband and high-performance optoelectronic devices such as photodetectors, terahertz lasers, waveguides, and transparent electrodes [25]. Among these TIs, $\mathrm{Bi}_{2} \mathrm{Se}_{3}$ is a particularly interesting compound due to its relatively large bulk band gap and a simple surface state consisting of a single Dirac cone-like structure [26,27]. Study of 
the dielectric function reveals that the optical dielectric constant of $\mathrm{Bi}_{2} \mathrm{Se}_{3}$ can be very different for the trigonal and orthorhombic phases in the NIR regime [28]. $\mathrm{Bi}_{2} \mathrm{Se}_{3}$ exhibits a number of means through which their dielectric properties can be altered [28-33]. Herein, structural phase transition between trigonal and orthorhombic states, which is achieved by a high pressure and temperature, is proposed and studied as a means to change the intrinsic effective dielectric properties of the MDM-MMs [28].

Here, we numerically demonstrate a blueshift tunable nanometer-scale MM consisting of an elliptical nanohole array (ENA) embedded in the MDM multilayers where the dielectric core layer is a $\mathrm{Bi}_{2} \mathrm{Se}_{3}$ composite. Under a high pressure of 2 to $4.3 \mathrm{~Pa}$ at $500^{\circ} \mathrm{C}, \mathrm{Bi}_{2} \mathrm{Se}_{3}$ occurring in trigonal phase undergoes a transition to orthorhombic phase and features a large change in the values of the effective dielectric constant [28]. Accordingly, a massive blueshift of the resonant response (from 2,140 to 1,770 nm) of a $\mathrm{Bi}_{2} \mathrm{Se}_{3}$-based MDM-ENA is achieved in the NIR region. Our proposed blueshift tunable negative-index MM provides greater flexibility in the practical application and has a potential of enabling efficient switches and modulators in the NIR region.

\section{Methods}

The proposed MDM-ENA suspended in a vacuum is shown in Figure 1, with the coordinate axes and the polarization configuration of the normally incident light. The structure consists of trilayers of $\mathrm{Au} / \mathrm{Bi}_{2} \mathrm{Se}_{3} / \mathrm{Au}$. The thickness of each $\mathrm{Au}$ layer is $30 \mathrm{~nm}$, and the thickness of the $\mathrm{Bi}_{2} \mathrm{Se}_{3}$ layer is $60 \mathrm{~nm}$. The metamaterial parameters are optimized for the maximum sensitivity of the resonance to a change in the refractive index of the $\mathrm{Bi}_{2} \mathrm{Se}_{3}$ core dielectric layer in the NIR spectral range. The element resonator is shown in Figure 1b, where the pitch of the elliptical holes is $L=400 \mathrm{~nm}$, the diameters of the elliptical holes are $d_{1}=240 \mathrm{~nm}$ and $d_{2}=120 \mathrm{~nm}$, and $\beta$ is a cross-sectional plane of the structure. The $z$-axis is normal to the structure surface, and the $x-y$ plane is parallel to the structure surface. This simulated structure is periodically extended along the $x$ and $y$ axes. The tunable optical properties of the structure are calculated using 3D EM Explorer Studio [34], a commercial finite difference time domain (FDTD) code. In the simulation, a simple Drude-type model for $\mathrm{Au}$ permittivity was used, which is a good approximation to experimental values in the NIR region. The dielectric properties of $\mathrm{Au}$ as given by Johnson and Christy are used [35]. A plane wave source is simulated at normal incidence to the structure. The computational domain (400 $\mathrm{nm} \times$ $400 \mathrm{~nm} \times 1,000 \mathrm{~nm}$ ) has a perfectly matched layer (PML), absorbing boundaries in the $z$ direction and periodic boundaries in the $x-y$ plane [36]. A uniform FDTD mesh size is adopted. The mesh size is the same along all Cartesian axes: $\Delta x=\Delta y=\Delta z=2 \mathrm{~nm}$, which is sufficient to minimize the numerical errors arising from the FDTD method.

The topological insulator material $\mathrm{Bi}_{2} \mathrm{Se}_{3}$ was selected due to its significantly different optical properties between the trigonal and orthorhombic phases. The real $\left(\varepsilon_{1}\right)$ and imaginary $\left(\varepsilon_{2}\right)$ parts of the dielectric function for the different structural phases of $\mathrm{Bi}_{2} \mathrm{Se}_{3}$ were obtained from the published data in [28]; the NIR spectral region is shown in Figure 2. A large change in the dielectric function across the NIR is obtained after switching $\mathrm{Bi}_{2} \mathrm{Se}_{3}$ from trigonal to its orthorhombic phase.

After the complex coefficients of transmission $t=T_{a}$ $e^{i \phi_{a}}$ and reflection $r=R_{a} e^{i \phi_{r a}}$ are obtained by the 3D EM Explorer Studio, in which $T_{a}$ is the amplitude and $\phi_{a}$ is the phase of the transmission coefficient, and $R_{a}$ is the

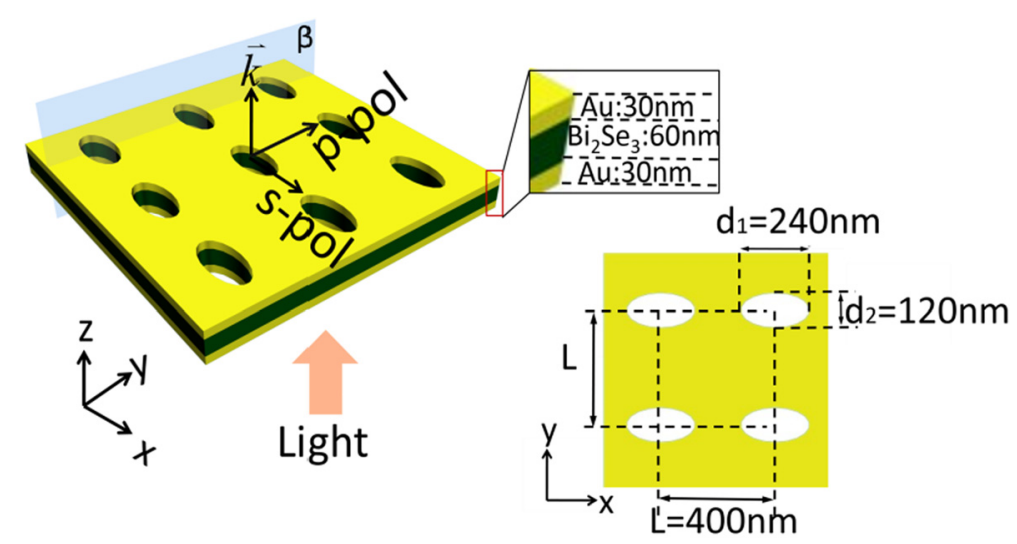

(a)

(b)

Figure 1 Schematic of the proposed structure. (a) Schematic of the MDM structure consisting of a 60-nm-thick $\mathrm{Bi}_{2} \mathrm{Se}_{3}$ dielectric layer between two 30-nm-thick Au films perforated with a square array of elliptical holes suspended in air. The lattice constant is $L=400 \mathrm{~nm}$, and hole diameters are $d_{1}=240 \mathrm{~nm}$ and $d_{2}=120 \mathrm{~nm}$. (b) Illustration of the square lattice of ENA. 

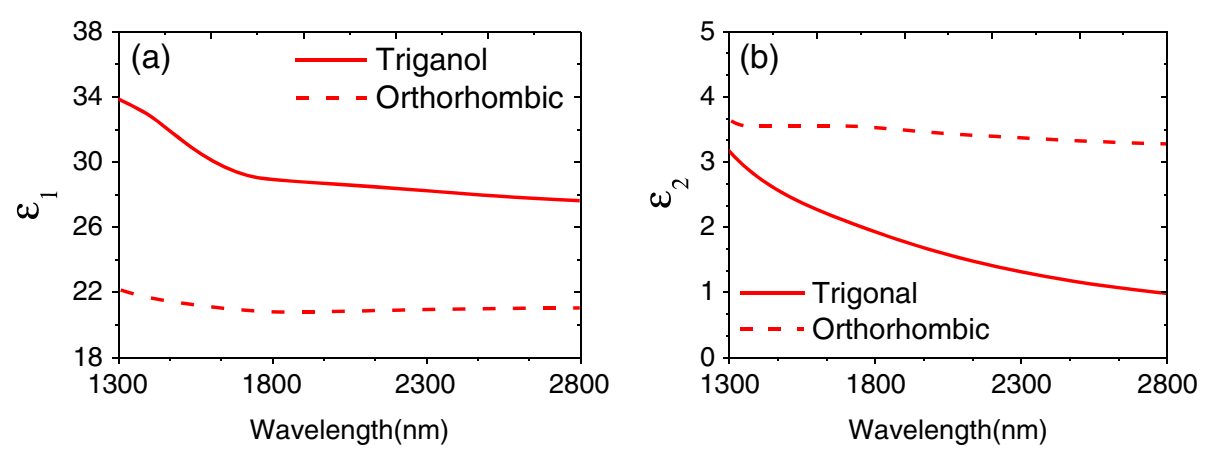

Figure 2 Dielectric constant of the $\mathrm{Bi}_{2} \mathrm{Se}_{3}$. (a) Real part of dielectric function $\varepsilon_{1}(\omega)$ for trigonal and orthorhombic phases of $\mathrm{Bi}_{2} \mathrm{Se}_{3}$. (b) Imaginary part of dielectric function $\varepsilon_{2}(\omega)$ for trigonal and orthorhombic phases of $\mathrm{Bi}_{2} \mathrm{Se}_{3}$.

amplitude and $\phi_{r a}$ is the phase of the reflection coefficient, the effective optical parameters can be extracted using the Fresnel formula [37].

For an equivalent isotropic homogenous slab of thickness $h$ surrounded by semi-infinite media with refractive index $n_{1}$ and $n_{3}$ under normal incidence, we have

$$
\begin{aligned}
& \eta= \pm \sqrt{\frac{(1+r)^{2}-t^{2}}{n_{1}^{2}(1-r)^{2}-n_{3}^{2} t^{2}}} \\
& n_{\text {eff }}= \pm \frac{1}{k h} \arccos \left[\frac{1}{t} \frac{n_{1}\left(1-r^{2}\right)+n_{3} t^{2}}{n_{1}+n_{3}+r\left(n_{3}-n_{1}\right)}\right]+\frac{2 \pi m}{k h} .
\end{aligned}
$$

The so-called material parameters $\varepsilon_{\text {eff }}$ and $\mu_{\text {eff }}$ of a single layer of a double-fishnet negative-index metamaterial are extracted using the well-known Nicholson-RossWeir (NRW) method [38-40]. Therefore, once $n_{\text {eff }}$ and $\eta$ are evaluated, the effective permittivity and permeability are calculated using

$$
\varepsilon_{\mathrm{eff}}=n_{\mathrm{eff}} / \eta, \quad \mu_{\mathrm{eff}}=n_{\mathrm{eff}} \eta
$$

where $n_{\text {eff }}$ is the effective refractive index, $\eta$ is the impedance, $h$ is the thickness of the structure, $k=\omega / c, c$ is the speed of light, $m$ is an arbitrary integer, and $n_{1}=$ $n_{3}=1$ since the structure is suspended in a vacuum. The signs of $n_{\text {eff }}$ and $\eta$ and the value of $m$ are resolved by the passivity of the metamaterial that requires the signs of the real part of impedance $\eta$ and imaginary part of effective index $n_{\text {eff }}$ to be positive, i.e., $\operatorname{Real}(\eta)>0$, Imag $\left(n_{\text {eff }}\right)>0$ which is consistent with the study described in $[39,40]$. We then apply this extraction approach to determine the change in the optical response of the structure when the phase of $\mathrm{Bi}_{2} \mathrm{Se}_{3}$ is switched between its trigonal and orthorhombic states.

\section{Results and discussion}

The ENA has a lower transmittance for $s$-polarized light due to the electric field's orientation with respect to the metallic stripe width [12]; hence, the polarization of the incident wave was set to be $p$-polarized. As shown in Figure 1a, $s$ polarization means that the incident electric field vector is parallel to the long axis of the ENA, and the incident electric field vector perpendicular to the long axis of the ENA is then denoted by $p$ polarization. We first investigate the transmittance $T=|t|^{2}$ and reflectance $R=|r|^{2}$ of the structure for $p$ polarization in Figure 3. Structures with a different dielectric constant of $\mathrm{Bi}_{2} \mathrm{Se}_{3}$ (shown in Figure 2) were modeled to investigate the effect of the phase change of $\mathrm{Bi}_{2} \mathrm{Se}_{3}$ on the position and amplitude of the spectrums. It can be seen that the resonance wavelength blueshifts from 2,140 to $1,770 \mathrm{~nm}$ when the structural phase of $\mathrm{Bi}_{2} \mathrm{Se}_{3}$ switches from trigonal to orthorhombic. The structure is impedance-matched, hence possessing a low reflectance corresponding to the dips in reflectance of Figure 3b for different forms of $\mathrm{Bi}_{2} \mathrm{Se}_{3}$.

In Figure 4, the transmission $(t)$ and reflection $(r)$ phases are demonstrated. The transmission phase exhibits a dip around the resonance, indicating that the light is advanced in phase at the resonance, characteristic of a left-handed material [41]. Importantly, changing the structural phase of the $\mathrm{Bi}_{2} \mathrm{Se}_{3}$ offers transmission and reflection phase tunability which implies tunable effective constitutive parameters in the structure.

Taking into account the subwavelength thickness of the structure, the extracted $n_{\text {eff }}$ can be retrieved from the transmission and reflection coefficients shown in Figure 5. For the MM with the trigonal $\mathrm{Bi}_{2} \mathrm{Se}_{3}$ dielectric layer, the negative-index band extends from 1,880 to $2,420 \mathrm{~nm}$ with a minimum value of the real part of the refractive index $\operatorname{Real}\left(n_{\text {eff }}\right)=-7$. Regarding losses, the figure of merit $(\mathrm{FOM})$ defined as $\mathrm{FOM}=\frac{\operatorname{Real}\left(n_{\text {eff }}\right)}{\operatorname{Imag}\left(n_{\text {eff }}\right)}$ is taken to show the overall performance of the MM, where $\operatorname{Imag}\left(n_{\text {eff }}\right)$ is the imaginary part of the refractive index. As shown in Figure 5c, the FOM for the trigonal phase is 2.7 at the operating wavelength of $2,080 \mathrm{~nm}$. The negative-index band of the orthorhombic $\mathrm{Bi}_{2} \mathrm{Se}_{3}$-based 

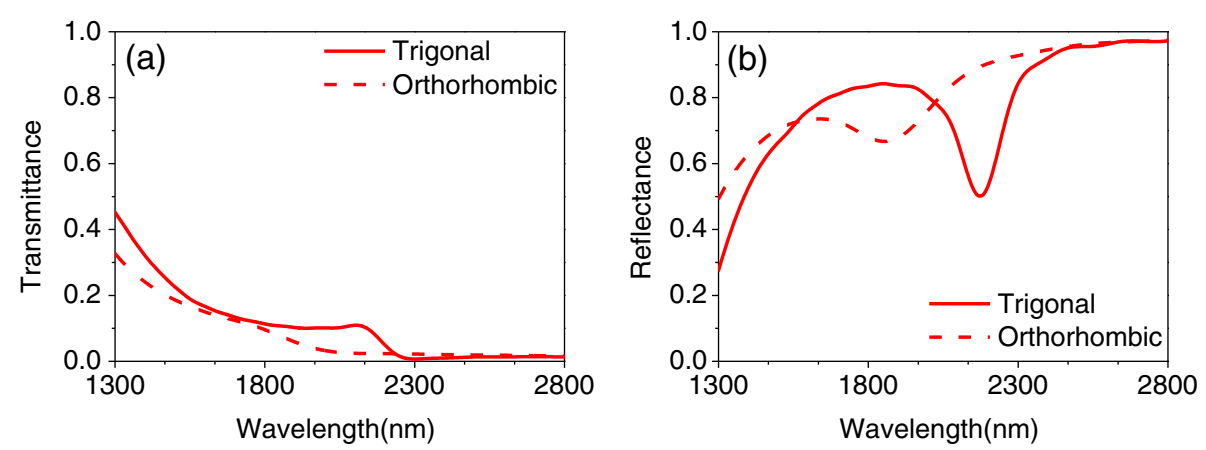

Figure 3 Transmittance and reflectance. 3D FDTD simulation of (a) spectrum of transmittance and (b) spectrum of reflectance, for the different phases of the $\mathrm{Bi}_{2} \mathrm{Se}_{3}$ dielectric layer, where the light source is $p$ polarization at normal incidence angle.

MM extends from 1,600 to 2,214 $\mathrm{nm}$ having a minimum value of $\operatorname{Real}\left(n_{\text {eff }}\right)=-3.2$. The FOM is 1.2 at the resonant wavelength of $1,756 \mathrm{~nm}$. Furthermore, the bandwidth of Real $\left(n_{\text {eff }}\right)$ becomes wider for the orthorhombic $\mathrm{Bi}_{2} \mathrm{Se}_{3}$ film in Figure 5a due to increased damping of the plasmon resonance but at the cost of an accompanied lower value of FOM in Figure 5c.

The refractive index is expressed in terms of the real and imaginary parts of the permeability $\mu_{\text {eff }}$ and permittivity $\varepsilon_{\text {eff. }}$ However, the sign of the real part of the permeability $\mu_{\text {eff: }}: \operatorname{Real}\left(\mu_{\text {eff }}\right)$ determines the relative magnitudes of the imaginary and real parts of the refractive index [41]. To achieve a negative index with a small loss, a negative $\operatorname{Real}\left(\mu_{\text {eff }}\right)$ is required. Therefore, we have simulated $\mu_{\text {eff }}$ and $\varepsilon_{\text {eff }}$ for the structure as shown in Figure 6. For the trigonal and orthorhombic phases of $\mathrm{Bi}_{2} \mathrm{Se}_{3}$, Real $\left(\mu_{\text {eff }}\right)$ has a Fano-type line shape and $\operatorname{Im}\left(\mu_{\text {eff }}\right)$ has a Lorentzian line shape in the region of the negative index. Moreover, a double-negative MM can be achieved when $\operatorname{Real}\left(\mu_{\text {eff }}\right)$ and $\operatorname{Real}\left(\varepsilon_{\text {eff }}\right)$ simultaneously reach negative values over a wide frequency range and thus a reduced loss. The maximum negative $\operatorname{Real}\left(\mu_{\text {eff }}\right)$ decreases with the phase transition from the trigonal to orthorhombic, hence resulting in the smaller value of the maximum negative $\operatorname{Real}\left(n_{\text {eff }}\right)$ at the orthorhombic phase.

This magnetic negative response can be explained looking at the current and field distribution at the resonance wavelengths. Figure 7 shows the current and total magnetic field intensity $H=\sqrt{\left|H_{x}\right|^{2}+\left|H_{y}\right|^{2}+\left|H_{z}\right|^{2}}$ for the magnetic resonant wavelengths of 2,140 and 1,770 $\mathrm{nm}$ at the $\beta$ plane shown in Figure 1. In the field maps of Figure 7, the arrows show the currents, whereas the color shows the intensity of the magnetic field. It clearly shows that the antiparallel currents are excited at opposite internal metallic interfaces, closed by an electric displacement current $J_{\mathrm{D}}$. Therefore, these virtual current loops between two Au layers on the $\beta$ plane give rise to magnetic resonant responses of negative $\operatorname{Re}\left(\mu_{\text {eff }}\right)$ that interact strongly with the incident magnetic field at which the total magnetic field intensity $H$ is strongly localized in the $\mathrm{Bi}_{2} \mathrm{Se}_{3}$ dielectric layer between the top and bottom Au layers [42].

Specifically, $H$ for the orthorhombic phase shown in Figure $7 \mathrm{~b}$ is weaker than the trigonal phase shown in Figure 7a. It depicts that the MM based on orthorhombic
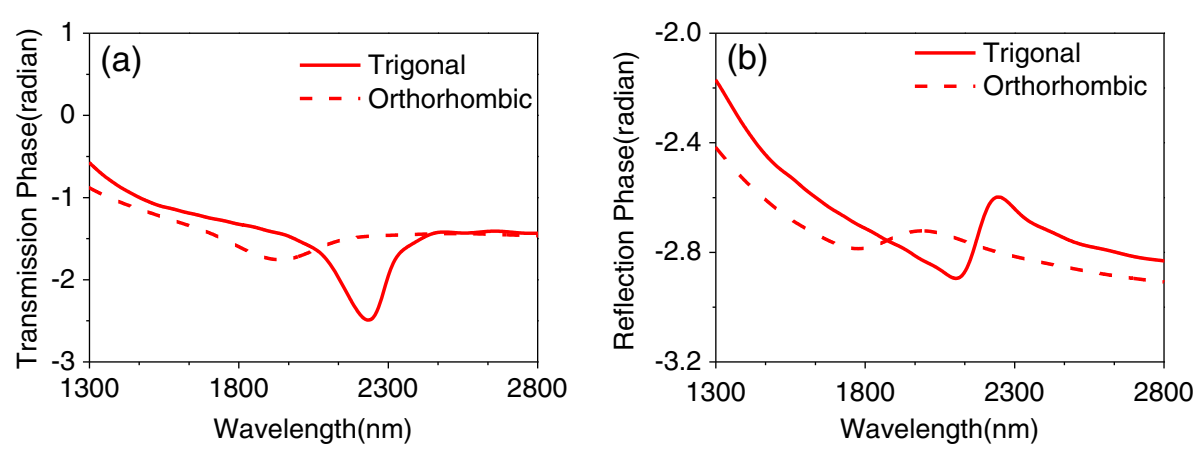

Figure 4 Transmission and reflection phase. 3D FDTD simulation of (a) phase of transmission and (b) phase of reflection, for the different phases of the $\mathrm{Bi}_{2} \mathrm{Se}_{3}$ dielectric layer, where the light source is $p$ polarization at normal incidence angle. 

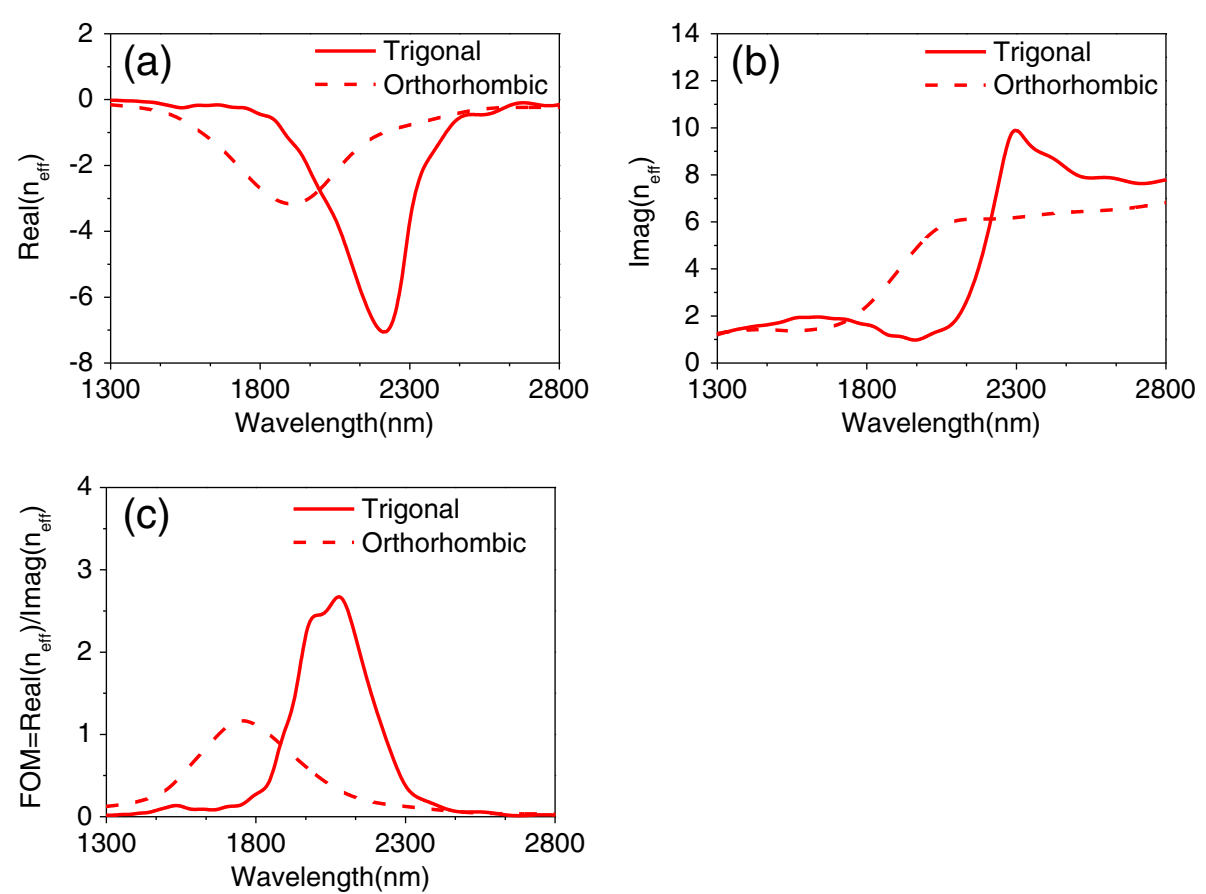

Figure 5 Effective index and figure of merit. 3D FDTD simulation of (a) real part of $n_{\text {eff, }}$ (b) imaginary part of $n_{\text {eff, }}$ and (c) figure of merit for the different phases of the $\mathrm{Bi}_{2} \mathrm{Se}_{3}$ dielectric layer, where the light source is $p$ polarization at normal incidence angle.
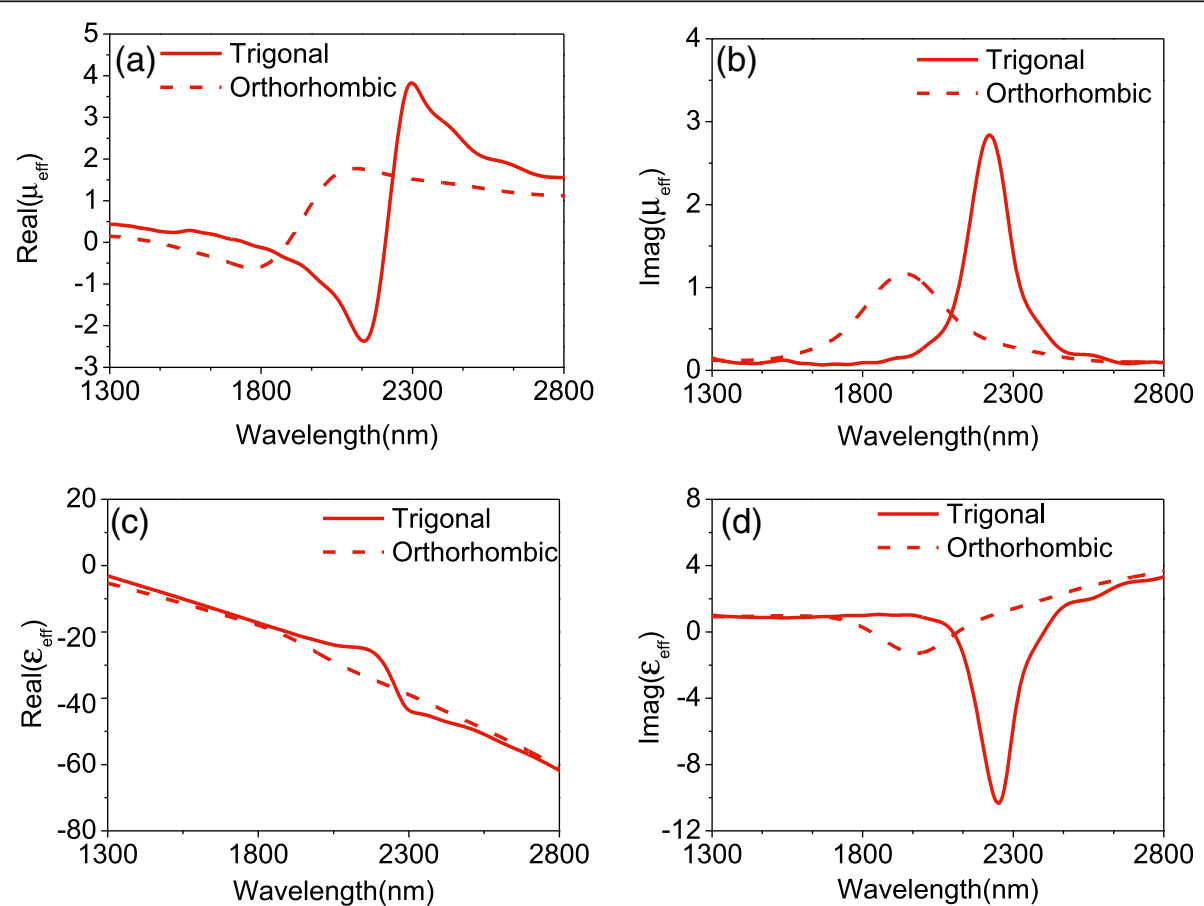

Figure 6 Permittivity and permeability. 3D FDTD simulation of (a) the real part of $\mu_{\text {eff }}$ (b) the imaginary part of $\mu_{\text {eff, }}$ (c) the real part of $\varepsilon_{\text {eff, }}$ and (d) the imaginary part of $\varepsilon_{\text {eff }}$ for the different phases of the $\mathrm{Bi}_{2} \mathrm{Se}_{3}$ dielectric layer, where the light source is $p$ polarization at normal incidence angle. 


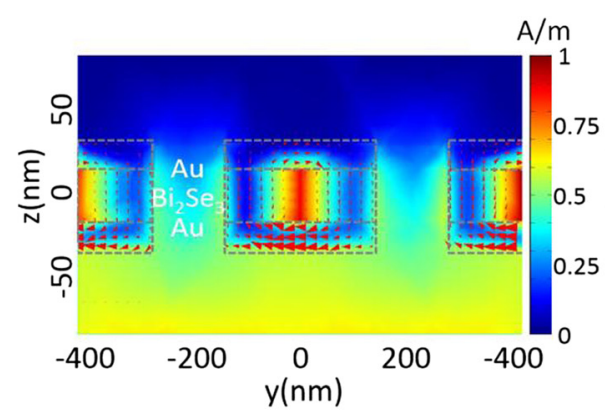

(a)

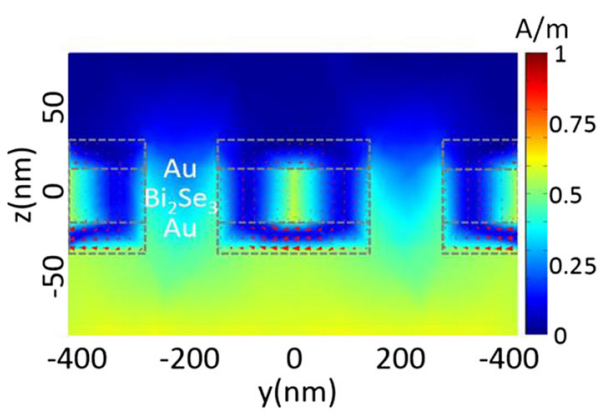

(b)

Figure 7 Magnetic field intensity and displacement current. A map of the normalized total magnetic field intensity distribution (color bar) and displacement current $\left(J_{D}\right)$ (arrows) along the plane $\beta$ : (a) at a 2,140-nm resonance wavelength for trigonal $\mathrm{Bi}_{2} \mathrm{Se}_{3}$ and $(\mathbf{b})$ at a $1,770-\mathrm{nm}$ resonance wavelength for orthorhombic $\mathrm{Bi}_{2} \mathrm{Se}_{3}$, where the light source is $p$ polarization at normal incidence angle.

phase has a smaller magnetic dipolar moment than the trigonal phase and thus smaller FOM.

To further understand the negative-index resonance in the metamaterials, it is useful to study the dispersion of the surface plasmon polariton (SPP) modes within the multilayer structure. Both the internal and external SPP modes in the multilayer metamaterials are similar to those of the same structure without resonant elements, i.e., MDM films [42], where the internal SPP mode resonates in the inner surfaces of the metal layers and the external SPP mode resonates in the outer surfaces of the metal layers. Therefore, the SPP dispersion relation of the multilayer metamaterial can be approximately approached by that of the MDM structure. In Figure 8, we
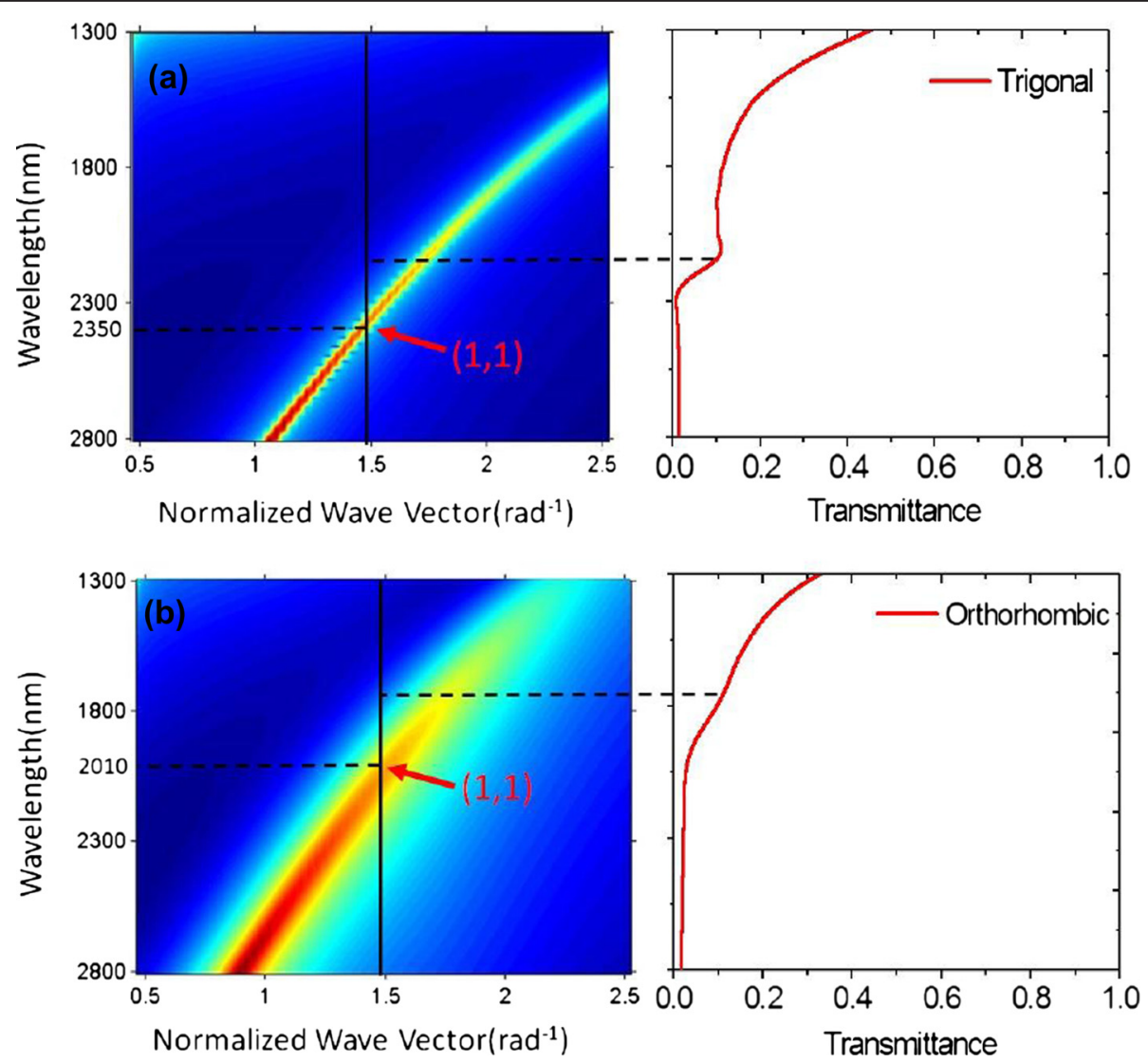

Figure 8 Dispersion relation of the structure. Representation of the dispersion relation of the $\mathrm{Au}_{-} \mathrm{Bi}_{2} \mathrm{Se}_{3}-\mathrm{Au}_{\mathrm{u}}$ trilayer (left) and the transmittance of the multilayer metamaterials (right) for both (a) trigonal $\mathrm{Bi}_{2} \mathrm{Se}_{3}$ and (b) orthorhombic $\mathrm{Bi}_{2} \mathrm{Se}_{3}$. 
have calculated the SPP mode dispersion relation of the $\mathrm{Au}-\mathrm{Bi}_{2} \mathrm{Se}_{3}-\mathrm{Au}$ sheets with the top $\mathrm{Au}$ film thickness $t_{1}=30 \mathrm{~nm}$, middle $\mathrm{Bi}_{2} \mathrm{Se}_{3}$ film thickness $t_{2}=60 \mathrm{~nm}$, and bottom $\mathrm{Au}$ film thickness $t_{3}=30 \mathrm{~nm}$. The transmittance spectrum of the multilayer metamaterials is also depicted together with the dispersion relation of the $\mathrm{Au}-\mathrm{Bi}_{2} \mathrm{Se}_{3}-\mathrm{Au}$ films.

Recalling the coupling condition from light to SPP modes [42], it can be seen that the $(1,1)$ internal resonance of the $\mathrm{Au}-\mathrm{Bi}_{2} \mathrm{Se}_{3}-\mathrm{Au}$ trilayer is excited at 2,350 nm associated with the trigonal $\mathrm{Bi}_{2} \mathrm{Se}_{3}$ in Figure 8a. This internal SPP resonance blueshifts to $2,010 \mathrm{~nm}$ when the trigonal state changes to the orthorhombic state as shown in Figure $8 \mathrm{~b}$. We also observe that the two internal $(1,1)$ modes which appear at 2,350 and 2,010 $\mathrm{nm}$ in the simple MDM structure do not perfectly match the two absorbance peaks at the resonance wavelengths of 2,140 and $1,770 \mathrm{~nm}$ in the multilayer metamaterials for both the trigonal and orthorhombic phases, respectively. This difference is because the dispersion relation of the SPP modes used as matching condition does not include the resonant squares, which cause a resonance shift [42].

\section{Conclusions}

In conclusion, this work numerically demonstrates the tunable optical properties of an ENA perforated through $\mathrm{Au} / \mathrm{Bi}_{2} \mathrm{Se}_{3} / \mathrm{Au}$ trilayers. We present that the MDM-ENA can be improved to exhibit a substantial frequency tunability of the intrinsic resonance in the NIR spectral region by selecting $\mathrm{Bi}_{2} \mathrm{Se}_{3}$ as the active dielectric material. Particularly, the resonant transmission, reflection, and the effective constitutive parameters of the $\mathrm{Bi}_{2} \mathrm{Se}_{3}$-coupled multilayer MM can be massively blueshifted by transiting the phase of the $\mathrm{Bi}_{2} \mathrm{Se}_{3}$ film from the trigonal to orthorhombic. This may offer an innovative and practical paradigm for the development of tunable photonic devices. We expect that our results will facilitate further experimental studies of the tunable MMs and make this technique suitable for tuning of plasmon resonance in the optical regime.

\section{Competing interests}

The authors declare that they have no competing interests.

\section{Authors' contributions}

TC conceived the idea of using topological insulator for tuning the resonance in the metamaterials, designed the metamaterial, and wrote the manuscript. SW carried out the simulations and prepared the figures. Both authors read and approved the final manuscript.

\section{Acknowledgements}

We acknowledge the financial support from National Natural Science Foundation of China (grant nos. 61172059, 51302026), PhD Programs Foundation of the Ministry of Education of China (grant no. 20110041120015), Postdoctoral Gathering Project of Liaoning Province (grant no. 2011921008), and The Fundamental Research for the Central University (grant no. DUT12JB01).
Received: 27 October 2013 Accepted: 3 December 2013

Published: 13 December 2013

\section{References}

1. Pendry JB: Negative refraction makes a perfect lens. Phys Rev Lett 2000, 61:3966-3969.

2. Qiu CW, Gao L: Resonant light scattering by small coated nonmagnetic spheres: magnetic resonances, negative refraction and prediction. J Opt Soc Am B 2008, 25:1728-1737.

3. Shalaev VM: Optical negative-index metamaterials. Nat Photonics 2007, $1: 41-48$

4. Soukoulis CM, Wegener M: Past achievements and future challenges in the development of three-dimensional photonic metamaterials. Nat Photonics 2011, 5:523-530.

5. Zheludev N: The road ahead for metamaterials. Science 2010, 328:5582-5583.

6. Zhou S, Huang X, Li Q, Xie YM: A study of shape optimization on the metallic nanoparticles for thin-film solar cells. Nanoscale Res Lett 2013, 8:447

7. Liaw JW, Chen HC, Kuo MK: Plasmonic Fano resonance and dip of $\mathrm{Au}-\mathrm{SiO}_{2}$-Au nanomatryoshka. Nanoscale Res Lett 2013, 8:468.

8. Zhang S, Fan W, Panoiu NC, Malloy KJ, Osgood RM, Brueck SRJ: Experimental demonstration of near-infrared negative-index metamaterials. Phys Rev Lett 2005, 95:137404

9. Li T, Li JQ, Wang FM, Wang QJ, Liu H, Zhu SN, Zhu YY: Exploring magnetic plasmon polaritons in optical transmission through hole arrays perforated in trilayer structures. Appl Phys Lett 2007, 90:251112.

10. Valentine J, Zhang S, Zentgraf T, Ulin-Avila E, Genov DA, Bartal G, Zhang X: Three-dimensional optical metamaterial with a negative refractive index. Nature 2008, 455:376-379.

11. Minovich A, Neshev DN, Powell DA, Shadrivov IV, Lapine M, Hattori HT, Tan HH, Jagadish C, Kivshar YS: Tilted response of fishnet metamaterials at near-infrared optical wavelengths. Phys Rev B 2010, 81:115109.

12. Zhang S, Fan W, Panoiu NC, Malloy KJ, Osgood RM, Brueck SRJ: Demonstration of metal-dielectric negative-index metamaterials with improved performance at optical frequencies. J Opt Soc Am B 2006, 23:434-438

13. Cao T, Cryan MJ: Study of incident angle dependence for dual-band double negative-index material using elliptical nanohole arrays. $J$ Opt Soc Am A 2012, 29:209-215.

14. Pendry JB, Holden A, Robbins D, Stewart W: Magnetism from conductors and enhanced nonlinear phenomena. IEEE Trans Microw Theory Tech 1999, 47(11):2075-2084.

15. Smith DR, Padilla WJ, Vier DC, Nemat-Nasser SC, Schultz S: Composite medium with simultaneously negative permeability and permittivity. Phys Rev Lett 2000, 84:4184-4187.

16. Zhao Q, Kang L, Du B, Li B, Zhou J, Tang H, Liang X, Zhang B: Electrically tunable negative permeability metamaterials based on nematic liquid crystals. Appl Phys Lett 2007, 90:011112.

17. Wang X, Kwon DH, Werner DH, Khoo IC, Kildishev AV, Shalaev VM: Tunable optical negative-index metamaterials employing anisotropic liquid crystals. Appl Phys Lett 2007, 91:143122.

18. Minovich A, Neshev DN, Powell DA, Shadrivov IV, Kivshar YS: Tunable fishnet metamaterials infiltrated by liquid crystals. Appl Phys Lett 2010, 96:193103.

19. Dicken MJ, Aydin K, Pryce IM, Sweatlock LA, Boyd EM, Walavalkar S, Ma J, Atwater HA: Frequency tunable near-infrared metamaterials based on $\mathrm{VO}_{2}$ phase transition. Opt Express 2009, 17:18330-18339.

20. Driscoll T, Kim HT, Chae BG, Kim BJ, Lee YW, Jokerst NM, Smith DR, Ventra MD, Basov DN: Memory metamaterials. Science 2009, 325:1518-1521.

21. Chen HT, O'Hara JF, Azad AK, Taylor AJ, Averitt RD, Shrekenhamer DB, Padilla WJ: Experimental demonstration of frequency-agile terahertz metamaterials. Nat Photon 2008, 2:295-298.

22. Hu XY, Zhang YB, Fu YL, Yang H, Gong QH: Low-power and ultrafast all-optical tunable nanometer-scale photonic metamaterials. Adv Mater 2011, 23:4295-4300.

23. Hasan MZ, Kane CL: Topological insulators. Rev Mod Phys 2010, 82:3045.

24. Qi XY, Zhang SC: Topological insulators and superconductors. Rev Mod Phys 2011, 83:1057.

25. Zhang $X$, Wang J, Zhang SC: Topological insulators for high-performance terahertz to infrared applications. Phys Rev B 2011, 82:245107. 
26. Hsieh D, Xia Y, Qian D, Wray L, Dil JH, Meier F, Osterwalder J, Patthey L, Checkelsky JG, Ong NP, Fedorov AV, Lin H, Bansil A, Grauer D, Hor YS, Cava RJ, Hasan MZ: A tunable topological insulator in the spin helical Dirac transport regime. Nature 2009, 460:1101.

27. Pan ZH, Vescovo E, Fedorov AV, Gardner D, Lee YS, Chu S, Gu GD, Valla T: Electronic structure of the topological insulator $\mathrm{Bi}_{2} \mathrm{Se}_{3}$ using angleresolved photoemission spectroscopy: evidence for a nearly full surface spin polarization. Phys Rev Lett 2011, 106:257004.

28. Sharma Y, Srivastava P: First-principles study of electronic and optical properties of $\mathrm{Bi}_{2} \mathrm{Se}_{3}$ in its trigonal and orthorhombic phases. AlP Conf Proc 2009, 1249:183-187.

29. Shao LH, Ruther M, Linden S, Essig S, Busch $K$, Weissmüller J, Wegener M: Electrochemical modulation of photonic metamaterials. Adv Mater 2010, 22:5173-5177.

30. Peng H, Dang W, Cao J, Chen Y, Wu D, Zheng W, Li H, Shen ZX, Liu Z: Topological insulator nanostructures for near-infrared transparent flexible electrodes. Nat Chem 2012, 4:281-286.

31. Dordevic SV, Wolf MS, Stojilovic N, Lei H, Petrovic C: Signatures of charge inhomogeneities in the infrared spectra of topological insulators $\mathrm{Bi}_{2} \mathrm{Se}_{3}$ $\mathrm{Bi}_{2} \mathrm{Te}_{3}$ and $\mathrm{Sb}_{2} \mathrm{Te}_{3}$. J Phys Condens Matter 2013, 25:075501.

32. Hafiz MM, El-Shazly O, Kinawy N: Reversible phase change in $\mathrm{Bi}_{x} \mathrm{Se}_{100-x}$ chalcogenide thin films for using as optical recording medium. App/ Surf Sci 2001, 171:231-241.

33. Zhao J, Liu H, Ehm L, Dong D, Chen Z, Gu G: High-pressure phase transitions, amorphization, and crystallization behaviors in $\mathrm{Bi}_{2} \mathrm{Se}_{3}$.J Phys Condens Matter 2013, 25:125602.

34. EM Explorer. http://www.emexplorer.net/.

35. Johnson PB, Christy RW: Optical constants of the noble metals. Phys Rev $B$ 1972, 6:4370-4379.

36. Berenger JP: Three-dimensional perfectly matched layer for the absorption of electromagnetic waves. J Comput Phys 1996, 127:363-379.

37. Born M, Wolf E, Bhatia AB: Principles of Optics. Cambridge: Cambridge University Press; 1997:61-70,

38. Nicolson AM, Ross GF: Measurement of the intrinsic properties of materials by time-domain techniques. IEEE Trans Instrum Meas 1970, 19:377-382.

39. Smith DR, Schultz $S$, Markos $P$, Soukoulis CM: Determination of effective permittivity and permeability of metamaterials from reflection and transmission coefficients. Phys Rev B 2002, 65:195104.

40. Chen XD, Grzegorczyk TM, Wu B, Pacheco JJ, Kong JA: Robust method to retrieve the constitutive effective parameters of metamaterials. Phys Rev E 2004, 70:016608.

41. Zhang S, Fan W, Malloy KJ, Brueck SRJ: Near-infrared double negative metamaterials. Opt Express 2005, 13:4922-4930.

42. Ortuño R, García-Meca C, Rodríguez-Fortuño FJ, Martí J, Martínez A: Role of surface plasmon polaritons on optical transmission through double layer metallic hole arrays. Phys Rev B 2009, 79:075425.

doi:10.1186/1556-276X-8-526

Cite this article as: $\mathrm{CaO}$ and Wang: Topological insulator metamaterials with tunable negative refractive index in the optical region. Nanoscale Research Letters 2013 8:526.

\section{Submit your manuscript to a SpringerOpen ${ }^{\circ}$ journal and benefit from:}

- Convenient online submission

- Rigorous peer review

- Immediate publication on acceptance

- Open access: articles freely available online

- High visibility within the field

- Retaining the copyright to your article

Submit your next manuscript at $\gg$ springeropen.com 